\title{
Conservation advances on Physalis peruviana L. and Spondia purpurea: a review
}

\author{
Juan Carlos DE LA VEGA ${ }^{1 *}$ (D), Valeria OLMEDO ${ }^{1}$, Clara Gabriela ORTEGA ${ }^{1}$, Marco Vinicio LARA ${ }^{1}$, \\ Rosario del Carmen ESPÍN ${ }^{1}$
}

\begin{abstract}
This research aims to describe the different methodologies that have been developed for the conservation of two fruits (Physalis peruviana L. and Spondia purpurea) present in the Ecuadorian highlands, as well as in other American countries. Information has been collected regarding the development of alternative and efficient technologies for the conservation of these fruits and their bioactive compounds. The studies performed to date are associated with the effect that the different conservation methods have shown in the post-harvest behavior (color, antioxidant potential, polyphenols, etc.) of the fruits, as well as its characterization in different maturation stages (soluble solids, acidity, $\mathrm{pH}$, etc.). The contrast and comparison of this information will support choosing the most appropriate methodology according to the objectives to be achieved and the available resources. The research concludes that the knowledge about the healthy components in fruits increase their consumption and therefore motivates, the research to preserve these components for a longer time, reducing costs and waste.
\end{abstract}

Keywords: food preservation; characterization; preservation effect.

Practical Application: The fruits in this research are substantially studied but there is yet some unknown ground.

\section{Introduction}

Among fruits valued by their consumers due to their health benefits, Physalis peruviana L. and Spondia purpurea have special relevance due to their polyphenolic content, ascorbic acid, and carotenoids among others, which provide antioxidant properties. Through the years, fruit production and consumption have increased. A 4.2\% annual growth was reported in South and Central America, while in Ecuador between 2013 and 2018 , goldenberry farming showed a growth of $10 \%$, with an increment of 160\% in its exportation from 2015 to 2016 (Moreno-Miranda et al., 2019).

This behavior brings up the need for carrying out research on the conservation of the fruit's properties, so sensorial and functional characteristics of the fruits can compete in international market. This review collects the latest advances in research about characterization and fruit conservation of Physalis peruviana L. and Spondia purpurea, with emphasis in their functional and nutritional properties depending on their maturity and postharvest conservation technology. Health benefits and the chronology of the conservation method on fruit properties were also included.

\subsection{Characterization}

Among the most notorious factors that affect fruits physicochemical and functional properties are: farming zone, and edaphological and climate conditions to which the fruit is exposed. Many times, fruit properties are different even if they are of the same variety, as it is shown in this paper. Physalis peruviana L. from Argentina (Bazalar Pereda et al., 2019), Colombia (Bravo \& Osorio, 2016), Peru (Maruenda et al., 2018), Brazil (Licodiedoff et al., 2016), South Africa (Carvalho et al., 2015), Egypt, South Africa, India, New Zeeland, Australia and Great Britain (Ramadan et al., 2015) have been reported. But among countries, the most advanced research is found in Colombia, where a normative (Norma Técnica Colombiana, 1999) to identify fruit maturity according to its color has been developed.

The research spectrum for Spondia purpurea is not extensive, and most observations are performed in Brazil (da Silva et al., 2016; Solorzano-Morán et al., 2015) and Mexico (Álvarez-Vargas et al., 2017), even when the farming region covers Venezuela, Colombia, Ecuador, Peru and Central America. Most studies have been focused on functional, nutritional and physicochemical characterization, and the application of conservation technology. Due to differences in data, the information on properties will be compared in tables for easier interpretation. Table 1 shows proximate analysis $(\mathrm{g} / 100 \mathrm{~g}$ fresh weight) and physicochemical properties such as $\mathrm{pH}$, acidity (\%w/w citric acid), soluble solids (SS), humidity, and maturity indices (MI).

Table 1 shows MI, they are different so are their properties, this factor is the most relevant to characterize a fruit. Table 2 shows mineral $(\mathrm{mg} / 100 \mathrm{~g}$ fresh weight) and the functional content of Physalis peruviana L. as vitamin A, C, carotenoids, polyphenols, and antioxidant activity (aa). These last properties are widely studied, due to their relevance; aa is expressed as 
$\mu \mathrm{mol}$ Trolox/g fresh weight, vitamin A as mg equivalent retinol activity (ERA)/100 g fresh weight (fw), vitamin C as mg acid ascorbic/100 $\mathrm{g}$ fw and polyphenols as mg gallic acid equivalent (EGA)/100 g fw.

Table 1. Physicochemical characteristics and proximate analysis of Physalis peruviana L.

\begin{tabular}{lcc}
\hline \multicolumn{1}{c}{ Property } & Muñoz et al. (2017) & $\begin{array}{c}\text { Bazalar Pereda et al. } \\
(2019)\end{array}$ \\
\hline $\mathrm{pH}$ & $3.947 \pm 0.012$ & $3.92 \pm 0.03$ \\
Acidity & $0.943 \pm 0.030$ & $1.85 \pm 0.02$ \\
Soluble solids, SS ( ${ }^{\circ}$ Brix $)$ & $13.40 \pm 0.10$ & $14.80 \pm 0.10$ \\
Humidity (\%) & - & $79.11 \pm 1.24$ \\
Maturity indices, MI & 14.4 & 8.00 \\
Carbohydrates & - & $14.22 \pm 0.69$ \\
Protein & - & $1.35 \pm 0.05$ \\
Lipids & - & $0.39 \pm 0.01$ \\
Fiber & - & $4.12 \pm 0.10$ \\
Ash & - & $0.81 \pm 0.03$ \\
\hline
\end{tabular}

Table 2. Mineral and functional content of Physalis peruviana L.

\begin{tabular}{lcc}
\hline \multicolumn{1}{c}{ Property } & $\begin{array}{c}\text { Olivares- } \\
\text { Tenorio et al. } \\
(2016)\end{array}$ & $\begin{array}{c}\text { Bazalar Pereda et al. } \\
(2019)\end{array}$ \\
\hline $\mathrm{K}$ & $55.3-501.9$ & $373.25 \pm 15.50$ \\
$\mathrm{Mg}$ & $34.7-120.1$ & $48.7 \pm 2.40$ \\
$\mathrm{Na}$ & 52.7 & $8.78 \pm 0.40$ \\
$\mathrm{Ca}$ & $7.0-37.7$ & $11.17 \pm 0.50$ \\
$\mathrm{Cu}$ & 0.7 & $0.35 \pm 0.02$ \\
Mn & 0.7 & - \\
Fe & $0.1-3.9$ & - \\
$\mathrm{P}$ & $34.0-54.9$ & $1.24 \pm 0.06$ \\
Carotenoids & $0-1100$ & $103.33 \pm 5.01$ \\
Vitamin A & - & $33.35 \pm 0.37$ \\
Vitamin C & $10-1000$ & \\
Polyphenols & 6.12 & 0.1112 \\
Antioxidant activity, aa & & \\
(2,2' - azino - bis(3- \\
ethylbenzothiazoline - 6
\end{tabular}

Mineral content depends on the farming zone, that is why a considerable variation on these parameters is expected. The wide range for functional properties could be attributed to different extraction and quantification methodologies, as it is known that extraction solution or quantification procedure can be modified from one author to another. This would affect data and so will the MI, climacteric fruits (as goldenberry) keep maturing and fluctuating functional components affecting aa.

There was characterization research on goldenberry either in juice or powder, where its functional properties were evaluated (Ordóñez-Santos et al., 2017), trying to maintain the original qualities, over different treatments (heat pasteurization or sonication). Table 3 shows properties of gooseberry juice without treatment.

Mexican plum (Spondia purpurea) does not have many studies but in Mexico and Brazil some of its physicochemical characteristics were described. It is known that among its functional properties has vitamin C, polyphenols, and carotenoids; the properties of the last ones are reflected on the epidermis. It possesses a high senescence rate, that is, in a few days it is no longer suitable for consumption. Research on conservation is increasing by trying to preserve its properties or studying new alternatives of consumption while, maintaining its sensorial, nutritional, and functional properties (Muñoz-López et al., 2018). Table 4 shows physicochemical and functional properties of Spondia purpurea.

Physalis peruviana L. and Spondia purpurea have benefits for health consumers due to their aa. The increase in cardiovascular diseases in recent decades (World Health Organization, 2017) has also boosted interest in foods that contain bioactive compounds with aa. These health problems are mostly caused by oxidative stress, due to oxygen reactive species (ORS) augmentation, produced by the oxidation of macromolecules with endothelial dysfunction, converting oxidative stress on a disbalance mediator between vasodilator and vasoconstrictor mechanism. It is also associated with chronic degenerative diseases such as cancer, diabetes, and premature aging. Functional components of goldenberry

Table 3. Properties of Physalis peruviana L. juice

\begin{tabular}{lc}
\hline \multicolumn{1}{c}{ Property } & Value \\
\hline $\mathrm{L}^{*}$ & $37.73 \pm 0.74$ \\
Chrome & $20.55 \pm 0.75$ \\
Hue $\left(^{\circ}\right)$ & $83.14 \pm 2.50$ \\
$\mathrm{pH}$ & $3.98 \pm 0.18$ \\
Titrable acid $(\mathrm{g}$ citric acid/100 $\mathrm{mL}$ juice) & $0.71 \pm 0.30$ \\
SS ( ${ }^{\circ}$ Brix $)$ & $6.82 \pm 0.70$ \\
Total phenol content $(\mu \mathrm{g}$ EGA/g juice) & $801.45 \pm 10.91$ \\
Ascorbic acid $(\mathrm{mg} / 100 \mathrm{~mL})$ & $17.17 \pm 0.80$ \\
\hline
\end{tabular}


Table 4. Physicochemical and functional properties of Spondia purpurea.

\begin{tabular}{|c|c|c|c|}
\hline Property & Maldonado-Astudillo et al. (2014) & Solorzano-Morán et al. (2015) & Álvarez-Vargas et al. (2017) \\
\hline $\mathrm{L}^{*}$ & - & 40.88 & $43.93 \pm 7.65$ \\
\hline Chroma & - & 43.17 & $36.77 \pm 14.71$ \\
\hline $\mathrm{Hue}^{\circ}$ & - & 42.86 & $43.55 \pm 23.51$ \\
\hline $\mathrm{pH}$ & $2.6-6$ & - & - \\
\hline Tritable acidity (\%) & $0.01-1.98$ & - & $0.23 \pm 0.10$ \\
\hline SS ( ${ }^{\circ}$ Brix) & $3.2-2.7$ & 16.65 & $16.87 \pm 4.16$ \\
\hline Polyphenols ( $\mu \mathrm{g}$ EGA/g fw) & $1000-2300$ & $500-3000$ & 420.73 \\
\hline Carotenoids ( $\mu \mathrm{g} / \mathrm{g}$ fw) & $171.2-243.79$ & $61-327$ & 197.84 \\
\hline Ascorbic acid (mg/100 g) & $7.36-88.1$ & $60-210$ & - \\
\hline $\begin{array}{l}\text { aa 2,2 - diphenyl - } 1 \text { - picrylhydrazyl, } \\
\text { DPPH (mg AAE/100 g) }\end{array}$ & $1.5 \mu \mathrm{M} / \mathrm{g}$ & $0.5-1.6 \mathrm{mM} \mathrm{AAE} / \mathrm{g}$ & 54.8 \\
\hline aa ABTS (mg AAE/100 g) & $6.25 \mu \mathrm{M} / \mathrm{g}$ & - & 127.9 \\
\hline aa FRAP (mg AAE/100 g) & - & - & 91.28 \\
\hline
\end{tabular}

and Mexican plum neutralize ORS, reducing its accumulation in cells and further damage.

It is known that of almost 40 carotenoids found on human blood, the most abundant are $\beta$-carotenes, lutein, zeaxanthin, lycopene, among others (Zimmer \& Hammond, 2007 cited by Eggersdorfer \& Wyss, 2018). $\beta$-carotene blocks low density lipoproteins oxidation and transforms into vitamin A (retinol). Lutein and zeaxanthin are known as macular pigments that absorb blue light, protecting retina from photochemical damage and neutralize ORS, so their consumption reduce macular degeneration risk (Barker et al., 2011 cited by Eggersdorfer \& Wyss, 2018).

The difference in mineral content between Spondia purpurea (Table 5) and Physalis peruviana (Table 2) is not significant, but it is observed that the variety of these minerals contributes to the osmotic balance of the cell, as well as to the proper functioning of enzymes that require these micronutrients.

\subsection{Storage methods comparation}

Massive production brought new conservation technics without specialized equipment, that is why there is a special emphasis in chitosan (Bautista-Baños et al., 2006 cited by Bautista-Baños et al., 2017). This is a semipermeable film which prolongs life of fruit and vegetable products with lower infection levels than any other technic, and this has been reported on Chitosan treated plums $(2.0$ and $2.5 \%)$ at $12{ }^{\circ} \mathrm{C}$. Table 6 shows a variety of experiments in the last years, conservation elements are specified so as process characteristics and fruit exposure conditions.
Table 5. Mineral content of Spondia purpurea (mg/100 g fw).

\begin{tabular}{ccc}
\hline Property & $\begin{array}{c}\text { Ramírez et al. (2008 cited } \\
\text { by Villarreal-Fuentes et al. } \\
(2019)\end{array}$ & $\begin{array}{c}\text { Koziol \& Macía (1998 } \\
\text { cited by Álvarez- } \\
\text { Vargas et al., 2017) }\end{array}$ \\
\hline $\mathrm{Ca}$ & $2.50-5.10$ & $6.00-25.00$ \\
$\mathrm{P}$ & $1.30-2.70$ & $32.00-56.00$ \\
$\mathrm{Fe}$ & $1.57-4.73$ & $0.09-1.22$ \\
$\mathrm{~K}$ & $6.20-24.00$ & $230.00-270.00$ \\
$\mathrm{Na}$ & - & $2.00-9.00$ \\
$\mathrm{Zn}$ & $1.03-1.77$ & 0.02 \\
$\mathrm{Mn}$ & $0.27-0.53$ & - \\
$\mathrm{Cu}$ & $0.43-0.97$ & - \\
$\mathrm{Mg}$ & $2.60-4.50$ & - \\
$\mathrm{N}$ & $4.00-9.50$ & - \\
\hline
\end{tabular}

The use of plastics of different densities remains as the best preservation method, despite researchers' questions and the appearance of smart packaging. Due to the easy acquisition and lower production cost of polyethylene packages, considering that storage conditions play a fundamental role in obtaining the best storage effectiveness, research shows that food has been kept in perfect condition for 15 days when combined with temperatures ranging between 10 degrees Celsius, it is also necessary to mention that light conditions to which these packages are exposed interfere with the shelf life. 
Table 6. Conservation mechanism.

\begin{tabular}{|c|c|c|c|}
\hline Conservation method & Characteristics & Conditions & Author \\
\hline Chitosan & Semipermeable film & $\begin{array}{c}2 \text { and } 2.5 \% \text { concentration } \\
12^{\circ} \mathrm{C}, 12 \text { days } \\
4{ }^{\circ} \mathrm{C}, 21 \text { days } \\
28^{\circ} \mathrm{C}, 6 \text { days }\end{array}$ & $\begin{array}{c}\text { Bautista-Baños et al. (2006 cited by } \\
\text { Bautista-Baños et al., 2017) } \\
\text { Muñoz et al. (2017) }\end{array}$ \\
\hline \multirow[t]{4}{*}{ Polystyrene } & Disinfected plastic & $13^{\circ} \mathrm{C}$ & $\begin{array}{l}\text { Vanegas ( } 2005 \text { cited by } \\
\text { Mohammed et al., 2019) }\end{array}$ \\
\hline & & Relative Humidity, RH 85-90\% & Maldonado-Astudillo et al. (2014 cited by \\
\hline & & Freeze $-20^{\circ} \mathrm{C}$ (immature fruits) & Marisco \& Pungartnik, 2015) \\
\hline & & $9.5^{\circ} \mathrm{C}$ (mature fruits) & Navarro et al. (2016) \\
\hline \multirow[t]{2}{*}{ PVC } & Film/recipient & $\begin{array}{l}23-25^{\circ} \mathrm{C} / 2-4 \text { days } \\
9-10^{\circ} \mathrm{C} / \text { until } 10 \text { days }\end{array}$ & Coltro et al. (2014) \\
\hline & & $\begin{array}{l}20^{\circ} \mathrm{C}, \mathrm{RH} 82 \% / 4 \text { days } \\
15^{\circ} \mathrm{C}, \mathrm{RH} 73 \% / 10 \text { days } \\
10^{\circ} \mathrm{C}, \mathrm{RH} 69 \% / 15 \text { days }\end{array}$ & $\begin{array}{l}\text { Maldonado-Astudillo et al. (2014 cited } \\
\text { by Marisco \& Pungartnik, 2015) }\end{array}$ \\
\hline Hermetic recipient & $\begin{array}{c}\text { In the presence or absence of } \\
\text { light and air }\end{array}$ & $\begin{array}{c}16^{\circ} \mathrm{C} \\
12 \text { Light hours/w/o light } \\
24 \text { Light hours/w/o light } \\
\text { Best condition } 14 \text { days w/o light }\end{array}$ & $\begin{array}{l}\text { Montalvo-Gonzalez et al. (2011 cited by } \\
\text { Mohammed et al., 2019) }\end{array}$ \\
\hline Freeze Dryer & Freeze/Lyophilization & $\begin{array}{l}20^{\circ} \mathrm{C} \text { and }-5^{\circ} \mathrm{C} \\
0.05 \text { and } 0.1 \mathrm{mbar}\end{array}$ & Muñoz-López et al. (2018) \\
\hline \multirow[t]{2}{*}{$\begin{array}{l}1 \text { - Methylcyclopropene (1 - } \\
\text { MCP) }\end{array}$} & $\begin{array}{l}\text { Synthesis vegetal regulator } \\
\text { acting as an ethylene action } \\
\text { inhibitor }\end{array}$ & $\begin{array}{c}\text { Dose: } 100,200,300 \mathrm{~nL} / \mathrm{L} \\
12 \text { hours, } 3 \text { days and ambient } \mathrm{T}\end{array}$ & $\begin{array}{l}\text { Osuna-García et al. (2011 cited by } \\
\text { Suárez-Vargas et al., 2017) }\end{array}$ \\
\hline & & $\begin{array}{c}22^{\circ} \mathrm{C} \pm 2{ }^{\circ} \mathrm{C}, 70 \pm 10 \% \mathrm{RH} \\
12 \text { days }\end{array}$ & $\begin{array}{l}\text { Osuna-García et al. (2011 cited by } \\
\text { Suárez-Vargas et al., 2017) }\end{array}$ \\
\hline $\begin{array}{l}\text { Ethylene and } \\
\text { 1-methylcyclopropene }\end{array}$ & Synthesis vegetal regulator & $20^{\circ} \mathrm{C}, \mathrm{RH} 75 \%, 11$ days & Balaguera-Lopez et al. (2017) \\
\hline Alginate $1 \%$ & Edible coating & $2{ }^{\circ} \mathrm{C}, 21$ days & Carvalho et al. (2015) \\
\hline Gelatin and calcium chloride & Edible coating & $\begin{array}{l}5{ }^{\circ} \mathrm{C}, 21 \text { days } \\
20^{\circ} \mathrm{C}, 14 \text { days }\end{array}$ & Licodiedoff et al. (2016) \\
\hline $\begin{array}{l}\text { Polyethylene terephthalate } \\
(\mathrm{PET}) \text { trays and } \\
\text { polypropylene (PP) baskets }\end{array}$ & Disinfected plastic & 4,8 and $12^{\circ} \mathrm{C}, \mathrm{RH} 80 \%$ & Olivares-Tenorio et al. (2017) \\
\hline Osmosis & Osmotic agents & $\begin{array}{c}18 \text { and } 25^{\circ} \mathrm{C} \text {, sucrose, mannitol, and } \\
\text { sorbitol }\end{array}$ & Rezende et al. (2018) \\
\hline
\end{tabular}

\section{Conclusion}

Technological developments have allowed to establish several preliminary tests to predict the physicochemical composition of the plum and goldenberry (in Mexico especially), which contributes significantly to the conservation methods used by making them more effective, optimizing the transfer times to the final consumer, and motivating the generation of new preservation alternatives; that through various research have shown better results of product storage at more favorable reproducibility conditions for the food industry, particularly those which work with large volumes of food worldwide.

\section{References}

Álvarez-Vargas, J. E., Alia-Tejacal, I., Chavez-Franco, S. H., Colinas-León, M. T., Nieto-Ángel, D., Rivera-Cabrera, F., \& Aguilar-Pérez, L. A.
(2017). Ciruelas Mexicanas (Spondias purpurea L.) de clima húmedo y seco: calidad, metabolitos funcionales y actividad antioxidante. Interciencia, 42(10), 653-660. Retrieved from https://www.redalyc. org/articulo.oa? $\mathrm{id}=33953313005$

Balaguera-Lopez, H. E., Espinal-Ruiz, M., Zacarias, L., \& Herrera, A. (2017). Effect of ethylene and 1-methylcyclopropene on the postharvest behavior of cape gooseberry fruits (Physalis peruviana L.). Food Science \& Technology International, 23(1), 86-96. http:// dx.doi.org/10.1177/1082013216658581. PMid:27440155.

Bautista-Baños, S., Ventura-Aguilar, R. I., Correa-Pacheco, Z., \& CoronaRangel, M. L. (2017). Chitosan: a versatile antimicrobial polysaccharide for fruit and vegetables in postharvest: a review. Revista Chapingo Serie Horticultura, 23(2), 103-121. http://dx.doi.org/10.5154/r.rchsh.2016.11.030.

Bazalar Pereda, M. S., Nazareno, M. A., \& Viturro, C. (2019). Nutritional and antioxidant properties of Physalis peruviana L. fruits from the argentinean northern andean region. Plant Foods for Human 
Nutrition, 74(1), 68-75. http://dx.doi.org/10.1007/s11130-018-07021. PMid:29335878.

Bravo, K., \& Osorio, E. (2016). Characterization of polyphenol oxidase from cape gooseberry (Physalis peruviana L.) fruit. Food Chemistry, 197, 185-190. http://dx.doi.org/10.1016/j.foodchem.2015.10.126.

Carvalho, C. P., Villaño, D., Moreno, D. A., Serrano, M., \& Valero, D. (2015). Alginate edible coating and cold storage for improving the physicochemical quality of cape gooseberry (Physalis peruviana L.). Journal of Food Science and Nutrition, 1, 102-112.

Coltro, L., Pitta, J. B., Costa, P. A., Fávaro Perez, M. Â., Araújo, V. A., \& Rodrigues, R. (2014). Migration of conventional and new plasticizers from PVC films into food simulants: a comparative study. Food Control, 44, 118-129. http://dx.doi.org/10.1016/j.foodcont.2014.03.058.

Eggersdorfer, M., \& Wyss, A. (2018). Carotenoids in human nutrition and health. Archives of Biochemistry and Biophysics, 652, 18-26. http:// dx.doi.org/10.1016/j.abb.2018.06.001. PMid:29885291.

Licodiedoff, S., Koslowski, L. A. D., Scartazzini, L., Monteiro, A. R., Ninow, J. L., \& Borges, C. D. (2016). Conservation of physalis by edible coating of gelatin and calcium chloride. International Food Research Journal, 23(4), 1629-1634. Retrieved from http://www. myjurnal.my/public/article-view.php?id=98511

Maldonado-Astudillo, Y. I., Alia-Tejacal, I., Núñez-Colín, C. A., Jiménez-Hernández, J., Pelayo-Zaldívar, C., Lopez-Martínez, V., Andrade-Rodríguez, M., Bautista-Baños, S., \& Valle-Guadarrama, S. (2014). Postharvest physiology and technology of Spondias purpurea L. and S. mombin L. Scientia Horticulturae, 174, 193-206. http:// dx.doi.org/10.1016/j.scienta.2014.05.016.

Marisco, G., \& Pungartnik, C. (2015). Spondias purpurea L. (Anacardiaceae): traditional uses, chemical composition and biological activities. Scientia Amazonia., 4(2), 10-18.

Maruenda, H., Cabrera, R., Cañari-Chumpitaz, C., López, J. M., \& Toubiana, D. (2018). NMR-Based metabolic study of fruits of Physalis peruviana L. grown in eight different peruvian ecosystems. Food Chemistry, 262, 94-101. http://dx.doi.org/10.1016/j.foodchem.2018.04.032. PMid:29751927.

Mohammed, M., Bridgemohan, P., Graham, O., Wickham, L., Bridgemohan, R. S. H., \& Mohammed, Z. (2019). Postharvest physiology, biochemistry and quality management of Chili Plum (Spondias purpurea var. Lutea): a review. Journal of Food Research, 8(3), 1-15. http://dx.doi.org/10.5539/jfr.v8n3p1.

Moreno-Miranda, C., Moreno-Miranda, R., Pilamala-Rosales, A. A., Molina-Sánchez, J. I., \& Cerda-Mejía, L. (2019). El sector hortofrutícola de Ecuador: principales características socio-productivas de la red agroalimentaria de la uvilla (Physalis peruviana). Ciencia Agricola, 16(1), 31-51. http://dx.doi.org/10.19053/01228420.v16.n1.2019.8809.

Muñoz, A., Barbosa, A., Bustos, D., Ramírez, Y., Vásquez, Y., García, J., \& Guancha, M. (2017). Conservación de uchuva (Physalis peruviana) mediante la aplicación de un recubrimiento a base de quitosano y áloe vera, utilizando el método de aspersión. Informado Técnico, 81(1), 86-94. http://dx.doi.org/10.23850/22565035.722.

Muñoz-López, C., Urrea-García, G. R., Jiménez-Fernández, M., Rodríguez-Jiménes, G. C., \& Luna-Solano, G. (2018). Efecto de las condiciones de liofilización en propiedades fisicoquímicas, contenido de pectina y capacidad de rehidratación de rodajas de ciruela (Spondias Purpurea L.). Agrociencia, 52, 1-13. Retrieved from http://www.scielo.org.mx/scielo.php?script=sci_arttext\&pid $=$ S1405-31952018000100001
Navarro, R., Pérez, M., García, C., Elvira, C., \& Gallardo, A. (2016). opening new gates for the modification of PVC or other PVC derivatives: synthetic strategies for the covalent binding of molecules to PVC. Polymers, 8(4), 152. http://dx.doi.org/10.3390/polym8040152. PMid:30979244.

Norma Técnica Colombiana - NTC. (1999). NTC 4580: Norma Técnica Colombiana sobre frutas frescas: uchuva: especificaciones. Bogotá, Colombia.

Olivares-Tenorio, M. L., Dekker, M., Verkerk, R., \& van Boekel, M. A. J. S. (2016). Health-promoting compounds in cape gooseberry (Physalis peruviana L.): review from a supply chain perspective. Trends in Food Science \& Technology, 57, 83-92. http://dx.doi. org/10.1016/j.tifs.2016.09.009.

Olivares-Tenorio, M.-L., Dekker, M., Van Boekel, M. A. J. S., \& Verkerk, R. (2017). Evaluating the effect of storage conditions on the shelf life of cape gooseberry (Physalis peruviana L.). Lebensmittel-Wissenschaft + Technologie, 80, 523-530. http://dx.doi.org/10.1016/j.lwt.2017.03.027.

Ordóñez-Santos, L. E., Martínez-Girón, J., \& Arias-Jaramillo, M. E. (2017). Effect of ultrasound treatment on visual color, vitamin C, total phenols and carotenoids content in cape gooseberry juice. Food Chemistry, 233, 96-100. http://dx.doi.org/10.1016/j. foodchem.2017.04.114. PMid:28530616.

Ramadan, M. M., El-Ghorab, A. H., \& Ghanem, K. Z. (2015). Volatile compounds, antioxidants, and cancer activities of Cape gooseberry fruit (Physalis peruviana L.): an in-vitro study. Journal of the Arab Society for Medical Research, 10(2), 56-64. http://dx.doi. org/10.4103/1687-4293.175556.

Rezende, R., Rodrigues, F., Rezende, R., Soares, J., Pasqual, M., \& Assis, F. (2018). In vitro conservation of Cape gooseberry through slowgrowth nodal segment cultures. Pesquisa Agropecuária Brasileira, 53(5), 651-655. http://dx.doi.org/10.1590/s0100-204x2018000500015.

Silva, Q. J., Figueiredo, F. J., \& Lima, V. L. (2016). Características físicas e químicas de cirigueleiras cultivadas na zona da Mata Norte de Pernambuco. Revista Ceres, 63(3), 285-290. http://dx.doi. org/10.1590/0034-737X201663030002.

Solorzano-Morán, S., Alia-Tejacal, I., Rivera-Cabrera, F., López-Martínez, V., Pérez-Flores, L. J., Pelayo-Zaldívar, C., Guillén-Sánchez, D., León-Sánchez, F. D., \& Maldonado-Astudillo, Y. I. (2015). Quality attributes and functional compounds of mexican plum (Spondias purpurea L.) fruit ecotypes. Fruits, 70(5), 261-270. http://dx.doi. org/10.1051/fruits/2015027.

Suárez-Vargas, A., Juárez-López, P., López-Martinez, V., Pérez Flores, L. J., Guillén Sánchez, D., \& Alia-Tejacal, I. (2017). Antioxidant activity and physicochemical parameters in 'cuernavaqueña' mexican plum (Spondias purpurea L.) At different ripening stages. Revista Brasileira de Fruticultura, 39(4), e-787. http://dx.doi. org/10.1590/0100-29452017787.

Villarreal-Fuentes, J. M., Alia-Tejacal, I., Pérez-Pérez, X. D., EspinosaZaragoza, S., Marroquín-Agreda, F. J., \& Núñez-Colín, C. A. (2019). Caracterización fisicoquímica de frutos de ciruela mexicana (Spondias purpurea L.) en el Soconusco, Chiapas. Ecosistemas Recursos Agropecuarios, 6(17), 219-229. http://dx.doi.org/10.19136/ era.a6n17.1949.

World Health Organization - WHO. (2017). Monitoreo de avances en materia de las enfermedades no transmisibles 2017. Ginebra: WHO Press. Retrieved from http://apps.who.int/iris/bitstream/hand le/10665/259806/9789243513027-spa.pdf?sequence $=1$ 